\title{
Application Accuracy of Automatic Registration in Frameless Stereotaxy
}

\author{
Jens Rachinger Boris von Keller Oliver Ganslandt Rudolf Fahlbusch \\ Christopher Nimsky
}

Department of Neurosurgery, University of Erlangen-Nürnberg, Erlangen, Germany

\section{Key Words}

Application accuracy $\cdot$ Intraoperative magnetic resonance imaging $\cdot$ Registration $\cdot$ Stereotaxy

\begin{abstract}
Objective: We compared the application accuracy of an infrared-based neuronavigation system when used with a novel automatic registration with its application accuracy when standard fiducial-based registration is performed. Methods: The automatic referencing tool is based on markers that are integrated in the headrest holder we routinely use in our intraoperative magnetic resonance imaging (MRI) setting and can be detected by the navigation software automatically. For navigation targeting we used a Plexiglas phantom with 32 notched rods of different heights. The phantom was fixed in the head holder and multiple optimized gradient echo slices containing the clamp-integrated markers were acquired. After that we measured a $T_{1}$ MPRAGE sequence with a slice thickness of $1.0 \mathrm{~mm}$ for navigation. The deepest points of the surface of the rods were defined as target points in image space. In three measurement series we referenced the phantom once with 4, once with 7 fiducials and twice automatically. In one series we performed only one automatic registration. The localization error was measured 3 times per rod and registration. Results: The median localization errors for standard registration with $7 \mathrm{fi}$ ducials were between 1.2 and $3.05 \mathrm{~mm}$. With 4 fiducials, me-
\end{abstract}

dians were in the range from 1.87 to $2.21 \mathrm{~mm}$. For the automatic registration we obtained median localization errors between 0.88 and $2.13 \mathrm{~mm}$. In 6 of the 8 samples that were compared the automatic registration showed an application accuracy that was highly significantly better ( $p<0.001$ in most cases) than that of fiducial-based standard registration. Conclusion: The application accuracy found for automatic referencing is at least not worse than that for standard registration no matter whether 4 or 7 fiducial markers were used. Therefore, its use in the operating room is feasible. In combination with intraoperative MRI it may become a favorable alternative to standard fiducial-based registration especially when an intraoperative update of navigation data is necessary.

Copyright $\odot 2006$ S. Karger AG, Basel

\section{Introduction}

In the field of neurosurgery stereotactic techniques are well known and have been widely used for a long time. In the context of stereotaxy, 'registration' basically means correlating extracranial markers to intracranial points and the history of stereotaxy has always been a history of registration, too. So, assuming a fixed relationship between extracranial landmarks and intracerebral structures, in 1908 Horsley and Clarke [1] developed a system for precise placement of intracerebral electrodes that was

\section{KARGER}

Fax +4161306 1234

E-Mail karger@karger.ch

www.karger.com
(C) 2006 S. Karger AG, Basel

$1011-6125 / 06 / 0843-0109 \$ 23.50 / 0$

Accessible online at:

www.karger.com/sfn
Jens Rachinger, MD

Department of Neurosurgery, University of Erlangen-Nürnberg

Schwabachanlage 6, DE-91054 Erlangen (Germany)

Tel. +49 9131853 3001, Fax +499131 8534569

E-Mail jens.rachinger@nch.imed.uni-erlangen.de 
fixed to landmarks of the skull of animals. In 1947 Spiegel and Wycis [2] created their stereotactic apparatus, which was the first one for the use in human beings. Leksell [3] as well as Talairach et al. [4] followed with their own systems. All these systems were based on a stereotactic frame mounted on the patient's head. 'Registration' was achieved by correlating frame-markers with intracranial structures seen on pneumoencephalograms and ventriculograms. The era of computed tomography led to a renaissance of stereotaxy accompanied by the development of new frame-based systems, for example by Goerss et al. [5], Brown [6], and Leksell and Jernberg [7]. With these systems abscesses can be drained, biopsies and functional neurosurgery can be performed. In an enhanced approach Kelly et al. [8] transposed volumetric information derived from CT imaging into stereotactic space. Thus, a method of computer-assisted stereotactic volumetric resection of intracranial tumors was created that showed its benefit in a number of studies [9-12]. But serious drawbacks remained: the devices are cumbersome to use and handicap open craniotomies. Furthermore, they have to be fixed to the patient's head invasively and do not allow interactive, image-guided surgery in real time. These facts and technical progresses, especially in computer science, at the end of the 1980s encouraged several research groups [13-15] to develop systems that work without the use of rigid frames and offer interactive, image-guided surgery in (near) real time, so-called frameless stereotaxy. These systems correlate physical space with image space (registration) in different ways. On the one hand, there are 'marker-fit' techniques using either extrinsic markers (self-adhesive or implanted in the skull bone) or anatomical landmarks for registration. On the other hand, there are 'surface-fit' techniques using the outer contour of the face and skull for the referencing process. Several studies [16-19] confirmed the reliability of these registration methods, and rapid progress was made towards automatic marker detection in image space and semiautomatic registration [20]. Nevertheless, the registration process itself still had to be performed manually and therefore remained time-consuming and prone to error. For this reason, a fast and reliable tool for automated registration is desirable. Besides registration, in the context of frameless stereotaxy 'accuracy' is another crucial issue. The phenomenon of intraoperative brain deformation caused by brain swelling, tumor removal, loss of CSF or insertion of retractors is known as 'brain shift'. This results in a considerable loss of accuracy. Without compensation for brain shift, neuronavigation systems cannot be trusted at critical steps of the surgical procedure
$[21,22]$. Brain shift can be reliably compensated for by updating the neuronavigation data with intraoperative image data [23]. From this follows that a registration method is needed that allows fast and safe intraoperative re-referencing using up-to-date intraoperative scans. The aim of this study was to develop and evaluate an automatic registration method that is based on integrated fiducial markers in a head holder that can be identified in pre- or intraoperative images automatically.

\section{Materials and Methods}

The automatic registration hardware, developed by BrainLab Inc., Heimstetten, Germany, in cooperation with our department, consists of a rigid geometrical distribution of 5 MRI markers within the MRI-compatible headrest holder we use in our intraoperative MRI setting [24] (fig. 1). The markers are spherical glass cells embedded in the headrest holder which are fixed by the MR marker support and contain an MRI contrast enhancement solution (gadolinium ratio 1:63, fig. 2). The contrast agent is sealed within the spherical volume with a double layer of silicone. The headrest holder provides two reproducible positions for the reference array ('left-feet' and 'left-head') that defines the origin of the coordinate system for navigation. For visualization of the 5 MRI markers, axial (thickness: $220 \mathrm{~mm}$, FoV: $300 \mathrm{~mm}$, scan time: $27 \mathrm{~s})$, coronal $(18 \mathrm{~mm}, 300 \mathrm{~mm}, 27 \mathrm{~s})$, and sagittal (25 mm, $300 \mathrm{~mm}, 33 \mathrm{~s}$ ) slices are acquired with an optimized gradient echo sequence (fig. 3,4$)$. After data transfer of the gradient echo sequences followed by one or multiple datasets for navigation, the navigation software automatically detects the navigation data with the MRI marker spheres and calculates the registration matrix. This matrix correlates image space to physical space linked by the reference array origin. Through a unique identifier referred to as 'frame of reference' all image datasets acquired during one imaging session, regardless of its modality such as $\mathrm{T}_{1^{-}}$or $\mathrm{T}_{2^{-}}$ weighted images, share the same virtual origin and thus can be used for navigation purposes.

This automatic registration method is based on the isometric match of a list of unsorted points. The relationship between the coordinate system of the acquired images (= virtual space) and the coordinate system of the patient or more specifically the coordinate system of the reference array used for navigation (= real space) is described by an affine transformation matrix. Here the affine transformation matrix describes the difference in translation and rotation between the virtual space and real space. In order to calculate the transformation matrix one can for example identify at least three points both in virtual space and in real space and try to match them optimally using translation and rotation. Using this automatic registration method a list of points for the virtual space is extracted from the dedicated gradient echo sequences in transversal, coronal and sagittal direction and matched with a list of corresponding points in real space. Since the slices created by the dedicated gradient echo sequences are rather thick in one dimension, the information of at least two of these sequences has to be combined to extract a valid point in virtual space. Assuming we just have a gradient echo sequence in transversal 


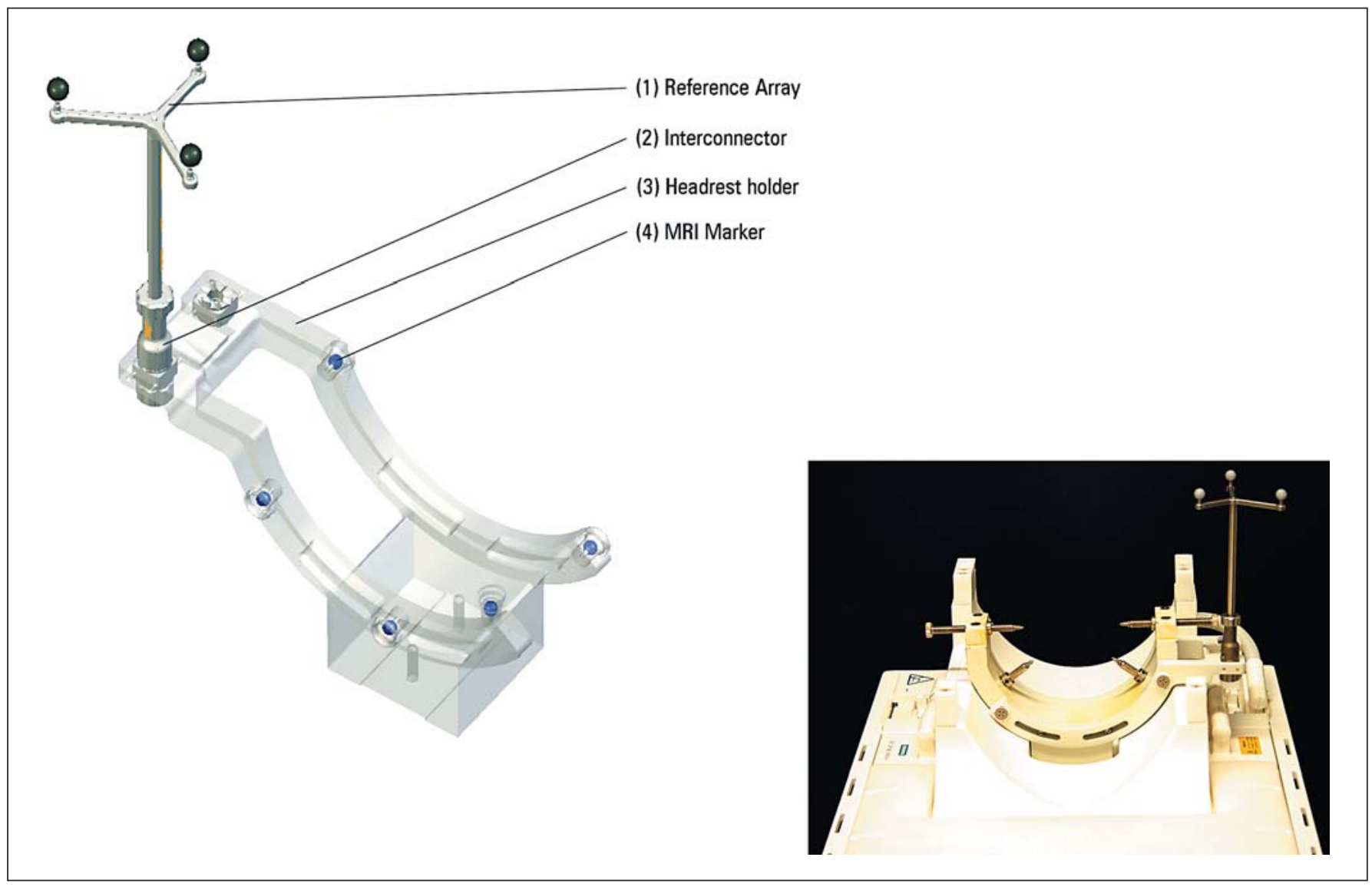

Fig. 1. Headrest holder with implemented MRI markers and reference array.

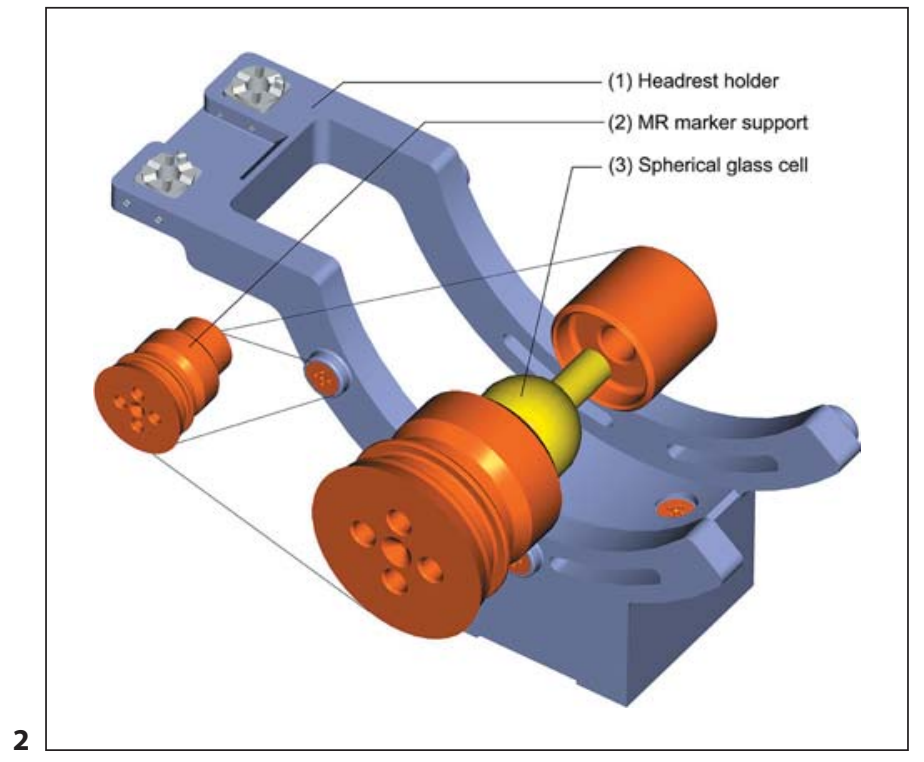

Fig. 2. MR markers within the headrest holder.

Fig. 3. Positioning of gradient echo sequence (GRE) slices for automatic registration.

Accuracy of Automatic Registration in Neuronavigation

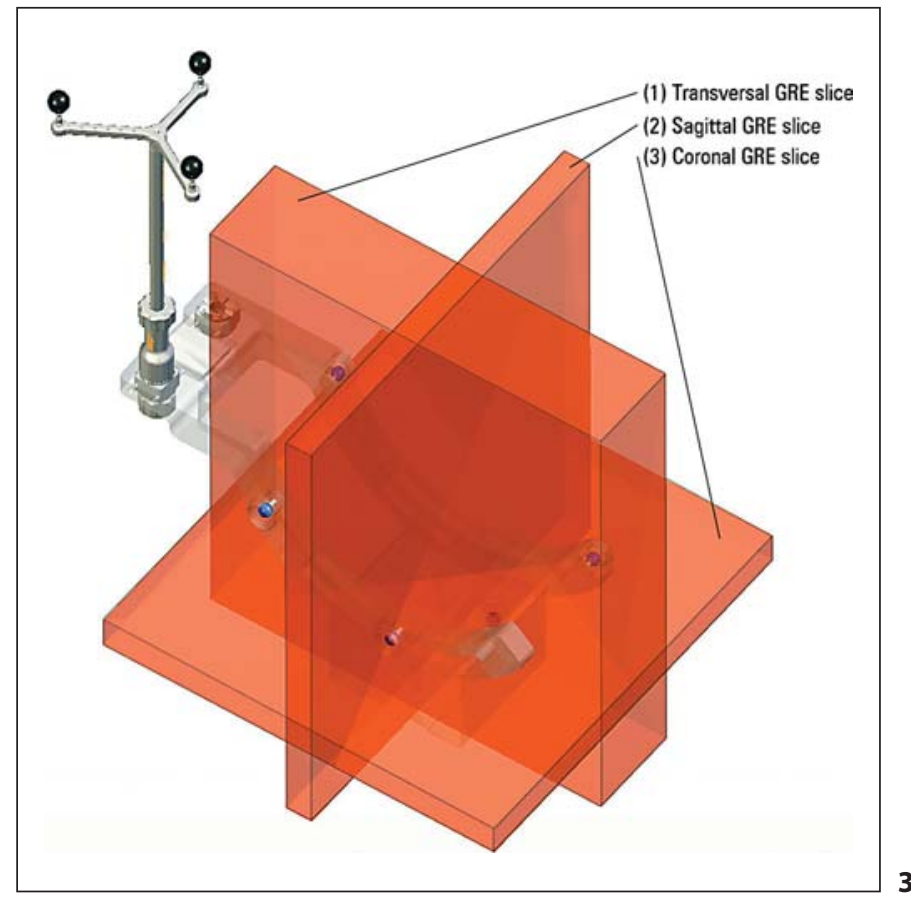

3 

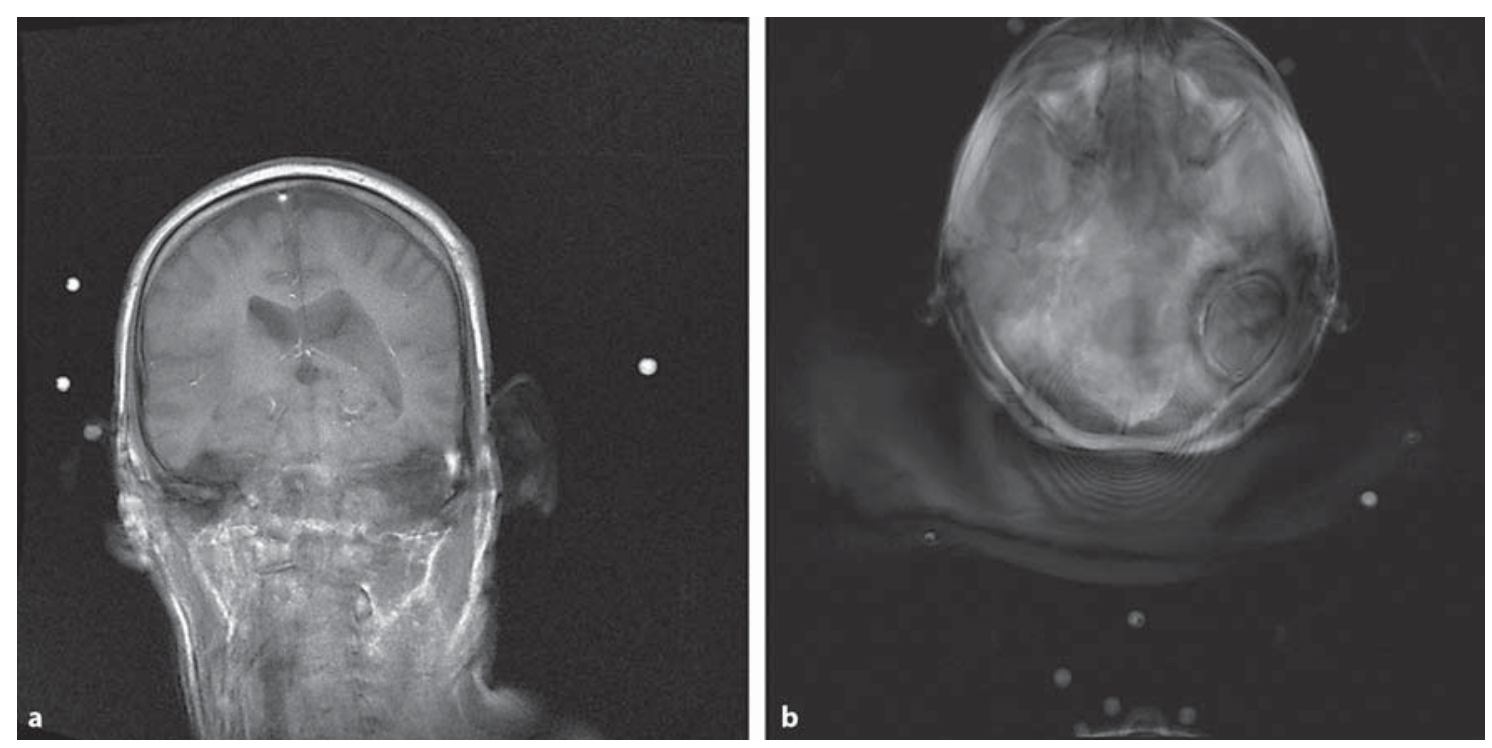

Fig. 4. Coronal (a) and axial (b) gradient echo sequence slice with patient's head.

direction, the spatial resolution would only be $0.60 \times 0.60 \times$ $80 \mathrm{~mm}$, hence the $\mathrm{z}$ - coordinate can only be determined to about $\pm 80 \mathrm{~mm}$. This is where the combination of at least one more dedicated gradient echo sequence is important, e.g. the coronal dimension with a spatial resolution of $0.60 \times 25 \times 0.60 \mathrm{~mm}$, where the inaccuracy lies in the $y$ component, but the $\mathrm{z}$ component has good spatial resolution. Identifying the same points in the transversal and coronal gradient echo images will result in an overall resolution of $0.60 \times 0.60 \times 0.60 \mathrm{~mm}$. The first step of the algorithm to extract valid points in virtual space is the automatic detection of the MRI marker spheres embedded in the headrest holder. Essentially, the automatic marker detection algorithm is a sophisticated image-processing function tuned to extract markers in the dimension of circles of known sizes in all slices of the dedicated gradient echo sequences. Then the correlation process of the detected MR markers takes place to combine the spatial information from the different gradient echo images. It is important to note that the position of the MRI marker spheres in the headrest holder was designed in that way that the identification of MR markers from the different dedicated gradient echo sequences is distinct. The last step is to find the optimal isometric match between the list of points extracted from the dedicated gradient echo sequences and the list of points of the MRI marker spheres in relation to the reference array, which again is well known according to the construction drawings of the headrest holder. By combining the information of different 'thick' slices as described above, scan time is reduced. Furthermore, a large area can be covered making it impossible to miss the MRI marker spheres in the imaging process. The user simply starts these dedicated sequences and does not have to worry about positioning.

The aim of our accuracy tests was to compare the application accuracy of the BrainLab VectorVision neuronavigation system (BrainLab Inc., Heimstetten, Germany) when used with the automatic registration (BrainLab internal denomination: iMRI

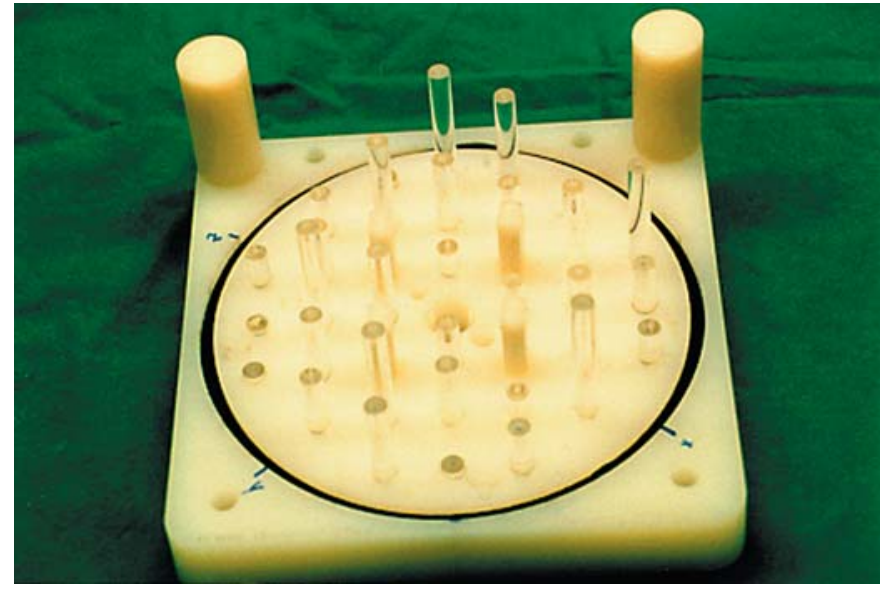

Fig. 5. Baseplate of the phantom with 32 Plexiglas rods used for navigation targeting.

TREGS S15T) with its accuracy when standard fiducial-based registration is performed. The study was designed to evaluate if the new tool provides accuracy and safety that are comparable to the standard method, thus allowing its clinical use in the operating room. For navigation targeting we used a Plexiglas phantom that we had utilized in a previous study [25]. The phantom consists of a square baseplate and a cylindrical cap which can be removed from the base (diameter: $168 \mathrm{~mm}$, height: $63 \mathrm{~mm}$ ). Thirtytwo rods of different heights with a funnel-shaped surface are mounted on the baseplate (fig. 5). The deepest point of the surface of each rod, i.e. the base of the superficial funnel with an approximate diameter of $1 \mathrm{~mm}$, was defined as the target point in image 


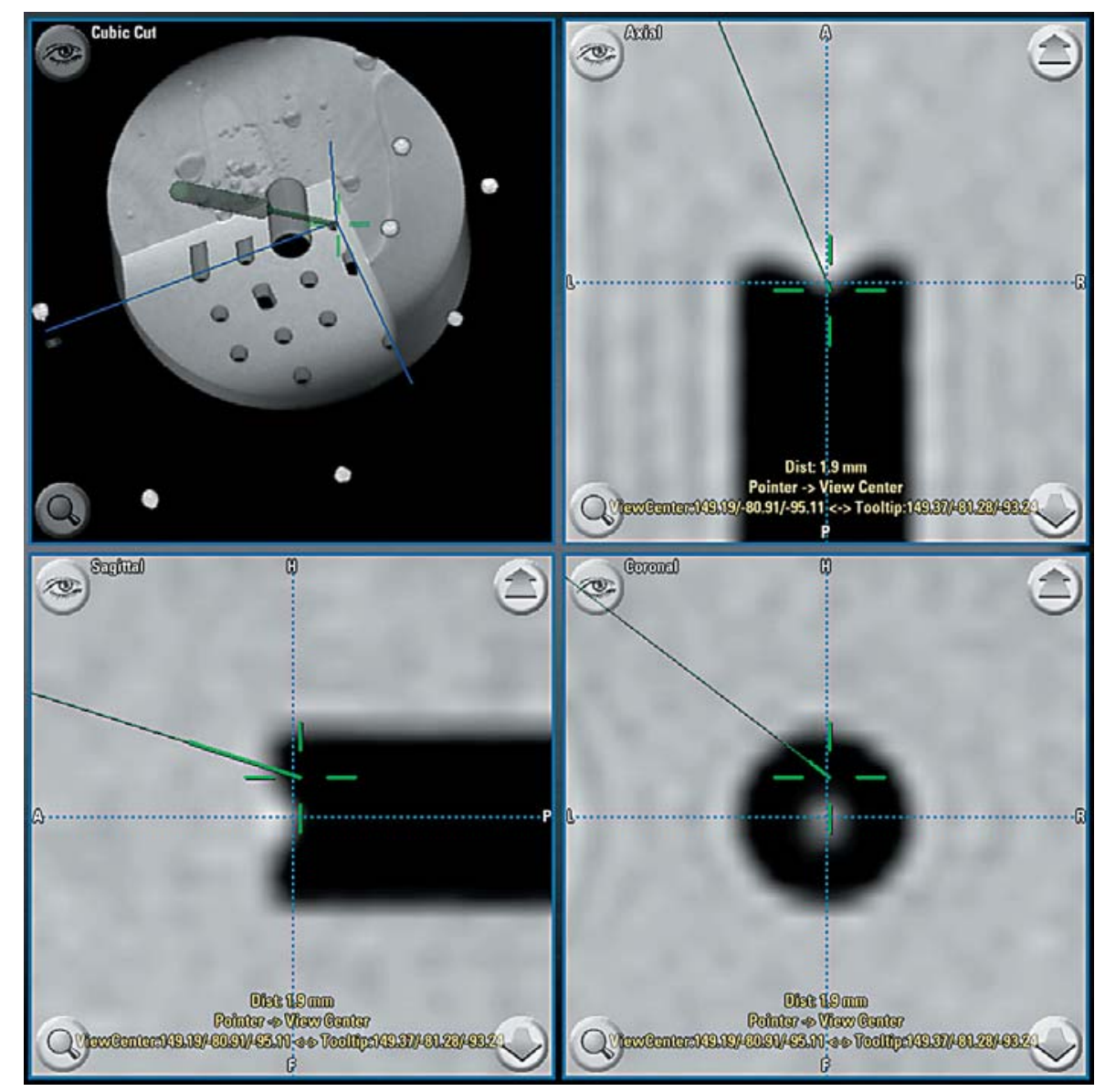

Fig. 6. Definition of target point in image space: the target point is represented by the deepest point of the surface of the Plexiglas rod. Oblique line depicting probe, in physical space centered on the deepest point of the surface of the corresponding rod and thus revealing the localization error.

space (fig. 6). When the transformation matrix was established, all measurement points were targeted with the pointer in a certain order. The pointer tool tip hereby represented the actual value with respect to the $(0 / 0 / 0)$ origin of the reference array. As soon as the pointer tool tip was positioned and the image map of the Plexiglas phantom rod appeared on the navigation screen (fig. 6), the freeze function was activated. The nominal value of the target point (the view center) was positioned exactly in the center of the deepest point of the funnel-shaped surface of the rod. Positioning was done by the manual alignment function of the image data set in the transversal, sagittal and coronal planes. On the screen the deviation (distance) was displayed as the Euclidean distance between the view center (nominal value) and the pointer tool tip (actual value) in millimeters. Finally the screenshot function was activated and the image map of the navigation screen was stored in the system. At the same time a log file containing the coordinates was created. For MR imaging the phantom was filled with a gadolinium water solution (ratio 1:200). Localization was based on a three-dimensional magnetic resonance dataset (MPRAGE; slice thickness, $1.0 \mathrm{~mm}$; FOV, $250 \mathrm{~mm}$; TR, 2,020 ms; TE, $4.38 \mathrm{~ms}$ ) which was acquired with a 1.5-tesla Magnetom Sonata Maestro class scanner (Siemens Medical Solutions, Erlangen, Germany). For standard registration 7 standard, self-adhesive fiducials were attached to the cylindrical cap of the phantom in a diffusely scat- tered pattern. The phantom itself was fixed tightly in the headrest holder, thus forming a rigid system. In total we performed four series of measurements, each with new MR datasets: in three of them we referenced the phantom once with 4 , once with 7 fiducials (reference array in 'left-feet' position) and twice automatically with 'TREGS' (reference array once in 'left-feet' position, once in 'left-head' position). In one series we did not reference automatically with the reference array in 'left-head' position. In each test series each target point was measured 3 times, thus obtaining a total number of error values of 1,440 (= 32 rods measured 3 times with 4 different registrations in three series plus 32 rods measured 3 times with 3 different registrations in one series). All localization error values were imported into two statistical software packages (Statgraphics Plus for Windows and SPSS 12.0 for Windows), thus allowing cross-checking of the results. The most important statistical values were calculated, and tests for determining the type of distribution as well as for comparing different samples were performed. Furthermore, we analyzed the spatial distribution of the error values over the phantom. 
Table 1. Most important descriptive values

\begin{tabular}{|c|c|c|c|c|c|c|c|}
\hline Registration & Series & $\begin{array}{l}\text { Mean } \\
\text { mm }\end{array}$ & $\begin{array}{l}\text { Median } \\
\text { mm }\end{array}$ & $\begin{array}{l}\mathrm{SD} \\
\mathrm{mm}\end{array}$ & $\begin{array}{l}\text { Min. } \\
\mathrm{mm}\end{array}$ & $\begin{array}{l}\text { Max. } \\
\text { mm }\end{array}$ & $\begin{array}{l}\text { 95th percentile } \\
\mathrm{mm}\end{array}$ \\
\hline Standard, 4 fiducials & 1 & 2.19 & 2.07 & 0.69 & 0.43 & 3.89 & 3.39 \\
\hline Standard, 7 fiducials & 1 & 1.48 & 1.34 & 0.55 & 0.57 & 2.80 & 2.55 \\
\hline Automated, left-feet ${ }^{1}$ & 1 & 1.00 & 0.96 & 0.38 & 0.25 & 2.04 & 1.85 \\
\hline Standard, 4 fiducials & 2 & 2.25 & 2.21 & 0.93 & 0.64 & 4.57 & 4.03 \\
\hline Standard, 7 fiducials & 2 & 3.01 & 3.05 & 0.92 & 0.0 & 4.94 & 4.33 \\
\hline Automated, left-feet ${ }^{1}$ & 2 & 0.99 & 0.88 & 0.48 & 0.15 & 2.53 & 1.85 \\
\hline Automated, left-head ${ }^{2}$ & 2 & 1.13 & 1.07 & 0.43 & 0.30 & 2.51 & 1.92 \\
\hline Standard, 4 fiducials & 3 & 1.88 & 1.87 & 0.80 & 0.21 & 3.79 & 3.20 \\
\hline Standard, 7 fiducials & 3 & 1.16 & 1.20 & 0.58 & 0.13 & 3.16 & 2.14 \\
\hline Automated, left-feet ${ }^{1}$ & 3 & 1.60 & 1.63 & 0.54 & 0.37 & 3.07 & 2.53 \\
\hline Automated, left-head ${ }^{2}$ & 3 & 1.44 & 1.42 & 0.40 & 0.69 & 2.94 & 2.19 \\
\hline Standard, 4 fiducials & 4 & 2.14 & 2.07 & 1.01 & 0.27 & 4.57 & 3.88 \\
\hline Standard, 7 fiducials & 4 & 2.56 & 2.36 & 1.02 & 0.0 & 4.73 & 4.02 \\
\hline Automated, left-feet ${ }^{1}$ & 4 & 2.11 & 2.13 & 0.44 & 0.83 & 3.04 & 2.71 \\
\hline Automated, left-head ${ }^{2}$ & 4 & 1.75 & 1.72 & 0.50 & 0.88 & 3.30 & 2.59 \\
\hline
\end{tabular}

${ }^{1}$ Reference array in 'left-feet' position.

${ }^{2}$ Reference array in 'left-head' position.

\section{Results}

The automatic registration could be established quickly and without any technical problems in each of the measurements. The most important statistical values are shown in table 1 . Chi-square as well as Shapiro-Wilks tests were performed to determine whether the samples come from a normal distribution. Twelve of the $15 \mathrm{sam}$ ples definitely did not fit a normal distribution, but showed a positively skewed distribution (example in fig. 7). Therefore, for comparison of the samples the Mann-Whitney (Wilcoxon) W test was used and $\mathrm{p}$ values less than 0.05 were regarded as statistically significant at the $95 \%$ confidence level. With the reference array in 'left-feet' position, in 6 of 8 compared registrations the automatic registration showed a significantly better application accuracy than standard registration, no matter whether 4 or 7 fiducials were used (table 2). In one comparison no significant difference was seen (fourth series, 4 fiducials vs. automatic referencing), whilst in the third series standard registration with 7 fiducials was superior to automated referencing. Changing the reference array to the 'left-head' position whilst leaving the 'left-feet' position for fiducial-based registration affected these results only minimally. The schematic representation of the localization error over the phantom revealed an almost identical pattern for all registration variations (represen-

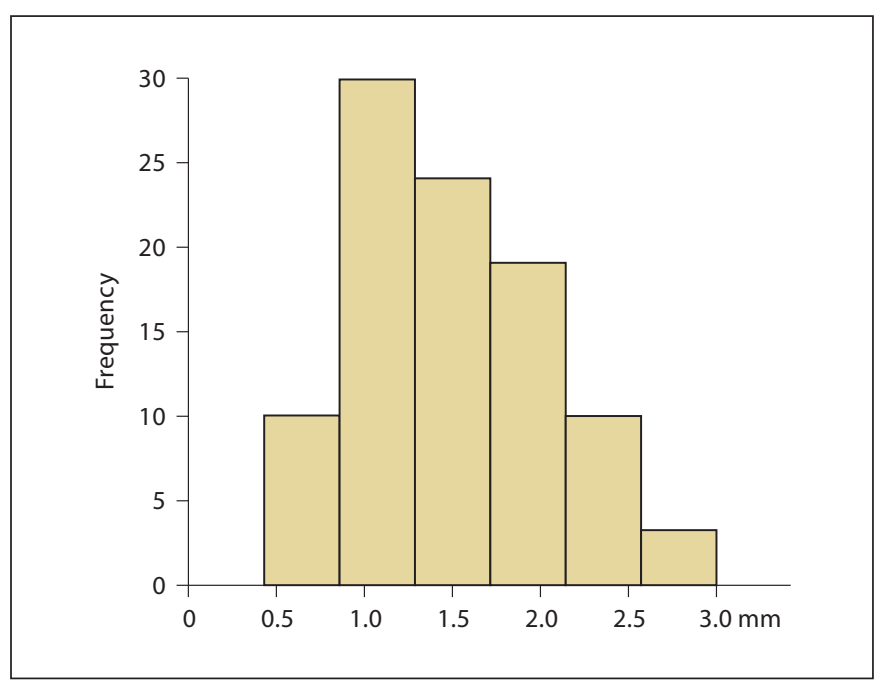

Fig. 7. Representative frequency histogram (standard registration with 7 fiducials).

tative example in fig. 8), the smallest localization error being found in the central areas of the phantom, especially in the left parts which are nearest to the reference array. By contrast, with growing distance from the center of the phantom localization error increases, especially in the posterior parts, which are the remotest ones from the camera. 
Table 2. Comparison between standard and automatic registration, Wilcoxon test for comparison of medians (for standard registration, reference array always in 'left-feet' position)

\begin{tabular}{llllr}
\hline $\begin{array}{l}\text { Se- } \\
\text { ries }\end{array}$ & $\begin{array}{l}\text { Standard } \\
\text { registration } \\
\text { with }\end{array}$ & $\begin{array}{l}\text { Automatic } \\
\text { registration } \\
\text { with refer- } \\
\text { ence array }\end{array}$ & Result & p value \\
\hline 1 & 4 fiducials & left-feet & automatic more accurate & $<0.001$ \\
1 & 7 fiducials & left-feet & automatic more accurate & $<0.001$ \\
2 & 4 fiducials & left-feet & automatic more accurate & $<0.001$ \\
2 & 7 fiducials & left-feet & automatic more accurate & $<0.001$ \\
2 & 4 fiducials & left-head & automatic more accurate & $<0.001$ \\
2 & 7 fiducials & left-head & automatic more accurate & $<0.001$ \\
3 & 4 fiducials & left-feet & automatic more accurate & 0.010 \\
3 & 7 fiducials & left-feet & standard more accurate & $<0.001$ \\
3 & 4 fiducials & left-head & automatic more accurate & $<0.001$ \\
3 & 7 fiducials & left-head & standard more accurate & $<0.001$ \\
4 & 4 fiducials & left-feet & no difference & \\
4 & 7 fiducials & left-feet & automatic more accurate & 0.002 \\
4 & 4 fiducials & left-head & automatic more accurate & 0.012 \\
4 & 7 fiducials & left-head & automatic more accurate & $<0.001$ \\
\hline
\end{tabular}

\section{Discussion}

Concerning neuronavigation, Steinmeier et al. [25] suggested defining the type of accuracy that is determined by imaging, technical accuracy of the system itself and registration as 'application accuracy'. Application accuracy combined with intraoperative factors such as brain shift or positional shift is defined as 'clinical accuracy'. In this study we wanted to evaluate a new tool for an automated registration process. Naturally, in this context intraoperative events have to be neglected and application accuracy is the technical feature to be tested. For this purpose we decided on a phantom study using a Plexiglas phantom. Compared to phantom studies investigating the application accuracy of frameless stereotactic systems by other groups [26-28], our results are in the same range for all relevant parameters, such as 95 th percentile, median and mean. This is true for the standard, fiducial-based registration as well as for the new automated process. Furthermore, in most of our measurements the automatic registration showed statistically significant lower localization errors than standard registration. Particularly with regard to the 95th percentile that is considerably lower in most cases, which is an important measure from a clinical point of view, there might even be relevant superiority over conventional registration. Only in the third measurement series the standard meth-

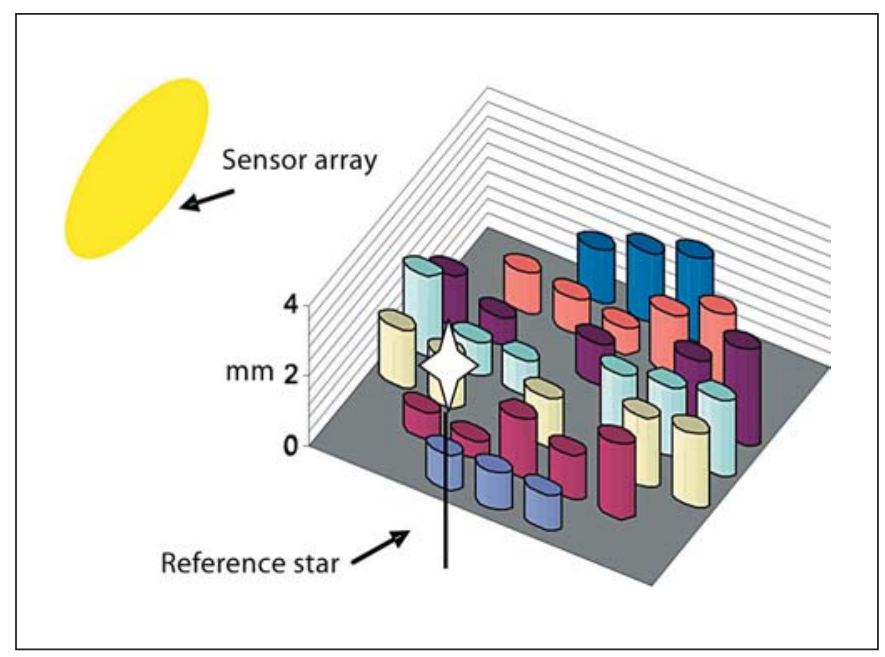

Fig. 8. Spatial distribution of localization errors over the phantom, each column representing one Plexiglas rod on its corresponding site on the baseplate (standard registration with 7 fiducials, average of all localization errors obtained with this configuration, example representative for all distributions). od using 7 fiducials yielded better results. In this series we observed an extraordinarily low localization error for this variation whilst the results for the automated process were fair. The exact reason for this phenomenon is not known, most probably we are dealing with an accidental, nonrepresentative effect. Another interesting phenomenon is the spatial heterogeneity of the distribution of localization errors over the phantom. As this spatial pattern is identical in all series independent of the type of registration, any influence of the distribution pattern of fiducials on this special effect is unlikely. Regarding the properties of the pattern and the whole setup, an influence of the distance of the target points to the reference array and to the camera seems possible. On the other hand, relevant spatial distortion of MR images is a wellknown phenomenon. The two main sources for this are gradient field nonlinearities (including 'barrel aberration', 'potato chip' and 'bow tie' effect) and resonance offsets (chemical shifts and magnetic field inhomogeneities) [29]. As the evaluation of geometric distortion in MR images was not part of this study, it was not assessed for the images used in the accuracy measurements. On the basis of our data a definitive statement as to whether the observed spatial heterogeneity of localization errors is caused by distorted MR images or the navigational system itself is therefore impossible. To answer this question, another study would be necessary. The occurrence of 
brain shift and the associated loss of accuracy is a serious limitation of the safe use of neuronavigation systems [21, 30]. So, several strategies for the detection and compensation of this phenomenon have been developed. Most of them are based on intraoperative images obtained by MR $[22,23]$, CT [31], or ultrasound [32, 33]. We favor the concept of intraoperative MR imaging and updating the frameless stereotactic system with these data $[21,23,24]$. Particularly, because MRI provides excellent, unsurpassed image quality, there is no X-ray contamination and, in the hands of the experienced worker, imaging can be performed safely and quickly. On the other hand, after image acquisition with the patient's opened skull under the sterile draping true re-referencing is difficult. Previously, intraoperative navigation updating necessitated the application of bone fiducials around the craniotomy opening, a time-consuming and cumbersome process [23]. The development of the automatic registration method (TREGS) described in the present study solved this problem. As the reference markers are integrated in the head holder and are detected automatically by the navigation software without the necessity of a process of pointing at extrinsic or intrinsic markers with an instru- ment or the focus point of the microscope, the special circumstances of an intraoperative situation no longer pose an obstacle for re-referencing and updating the navigation data.

\section{Conclusion}

Automatic registration is safe and reliable. The application accuracy found for the new tool is at least not worse than that for standard registration, its intraoperative use is therefore feasible. In combination with intraoperative MRI it may become a favorable alternative to standard, fiducial-based registration especially when an intraoperative update of the navigation data is needed.

\section{Acknowledgments}

We would like to thank BrainLab Inc. for the development of this automatic registration tool. For their constant help and support concerning the planning and the realization of the measurements we owe special thanks to the engineers Akos Dombay and Uli Mezger from BrainLab Inc.

\section{References}

1 Horsley V, Clarke RH: The structures and functions of the cerebellum examined by a new method. Brain 1908;31:45-124.

2 Spiegel EA, Wycis HAT, Marks M, Lee A: Stereotactic apparatus for operations on the human brain. Science 1947;106:349-350.

3 Leksell L: Stereotaxic apparatus for intracerebral surgery. Acta Chir Scand 1949;99: 229-233.

4 Talairach J, Hecaen M, David M, Monnier $\mathrm{M}$, Ajuriaguerra J: Recherches sur la coagulation therapeutique des structures souscorticales chez l'homme. Rev Neurol 1949; 81:4-24.

5 Goerss S, Kelly PJ, Kall B, Alker KJ Jr: A computed tomographic stereotactic adaptation system. Neurosurgery 1982;10:375-379.

6 Brown RA: A stereotactic head frame for use with CT body scanners. Invest Radiol 1979; 14:300-304.

7 Leksell D, Jernberg B: Stereotaxis and tomography: a technical note. Acta Neurochir $1980 ; 52: 1-7$.

8 Kelly PJ, Kall BA, Goerss S: Transposition of volumetric information derived from computed tomography scanning into stereotactic space. Surg Neurol 1984;21:465-471.
9 Camacho A, Kelly PJ: Volumetric stereotactic resection of superficial and deep seated intraaxial brain lesions. Acta Neurochir Suppl 1992;54:83-88.

10 Lyons MK, Kelly PJ: Computer-assisted stereotactic biopsy and volumetric resection of thalamic pilocytic astrocytomas: report of 23 cases. Stereotact Funct Neurosurg 1992; 59:100-104.

11 Kelly PJ, Kall BA, Goerss SJ: Results of computed tomography-based computer-assisted stereotactic resection of metastatic intracranial tumors. Neurosurgery 1988;22:7-17.

12 Kelly PJ: Volumetric stereotactic surgical resection of intra-axial brain mass lesions. Mayo Clin Proc 1988;63:1186-1198.

13 Watanabe E, Watanabe T, Manaka S, Mayanagi Y, Takakura K: Three-dimensional digitizer (neuronavigator): new equipment for computed tomography-guided stereotaxic surgery. Surg Neurol 1987;27:543-547.

14 Giorgi C, Luzzara M, Casolino DS, Ongania E: A computer controlled stereotactic arm: virtual reality in neurosurgical procedures. Acta Neurochir Suppl 1993;58:75-76.
15 Reinhardt HF, Meyer H, Amrein E: A computer-assisted device for the intraoperative CT-correlated localization of brain tumors. Eur Surg Res 1988;51-58.

16 Barnett GH, Miller DW, Weisenberger J: Frameless stereotaxy with scalp-applied fiducial markers for brain biopsy procedures: experience in 218 cases. J Neurosurg 1999;91: 569-576.

17 Wolfsberger S, Rossler K, Regatschnig R, Ungersbock K: Anatomical landmarks for image registration in frameless stereotactic neuronavigation. Neurosurg Rev 2002;25: 68-72.

18 Villalobos H, Germano IM: Clinical evaluation of multimodality registration in frameless stereotaxy. Comput Aided Surg 1999;4: 45-49.

19 Raabe A, Krishnan R, Wolff R, Hermann E, Zimmermann M, Seifert V: Laser surface scanning for patient registration in intracranial image-guided surgery. Neurosurgery 2002;50:797-801.

20 Kozak J, Nesper M, Fischer M, Lutze T, Goggelmann A, Hassfeld S, Wetter T: Semiautomated registration using new markers for assessing accuracy of a navigation system. Comput Aided Surg 2002;7:11-24. 
21 Nimsky C, Ganslandt O, Cerny S, Hastreiter P, Greiner G, Fahlbusch R: Quantification of, visualization of, and compensation for brain shift using intraoperative magnetic resonance imaging. Neurosurgery 2000;47: 1070-1079.

22 Nabavi A, Black PM, Gering DT, Westin CF, Mehta V, Pergolizzi RS Jr, Ferrant M, Warfield SK, Hata N, Schwartz RB, Wells WM 3rd, Kikinis R, Jolesz FA: Serial intraoperative magnetic resonance image of brain shift. Neurosurgery 2001;48:787-797.

23 Nimsky C, Ganslandt O, Hastreiter P, Fahlbusch R: Intraoperative compensation for brain shift. Surg Neurol 2001;56:406-407.

24 Nimsky C, Ganslandt O, von Keller B, Romstoeck J, Fahlbusch R: Intraoperative highfield-strength MR imaging: implementation and experience in 200 patients. Radiology 2004;233:67-78.
25 Steinmeier R, RachingerJ, Kaus M, Ganslandt O, Huk W, Fahlbusch R: Factors influencing the application accuracy of neuronavigation systems. Stereotact Funct Neurosurg 2000; 75:188-202.

26 Dorward NL, Alberti O, Palmer JD, Kitchen ND, Thomas DG: Accuracy of true frameless stereotaxy: in vivo measurement and laboratory phantom studies. Technical note. J Neurosurg 1999;90:160-168.

27 Zinreich SJ, Tebo SA, Long DM, Brem H, Mattox DE, Loury ME, van der Kolk CA, Koch WM, Kennedy DW, Bryan RN: Frameless stereotaxic integration of CT imaging data: accuracy and initial applications. Radiology 1993;188:735-742.

28 Lindseth F, Lango T, Bang J, Nagelhus Hernes TA: Accuracy evaluation of a 3D ultrasound based neuronavigation system. Comput Aided Surg 2002;7:197-222.

29 Sumanaweera TS, Adler JR, Napel S, Glover $\mathrm{GH}$ : Characterization of spatial distortion in magnetic resonance imaging and its implications for stereotactic surgery. Neurosurgery 1994;35:696-704.
30 Reinges MH, Nguyen HH, Krings T, Hutter BO, Rohde V, Gilsbach JM: Course of brain shift during microsurgical resection of supratentorial cerebral lesions: limits of conventional neuronavigation. Acta Neurochir 2004;146:369-377.

31 Nakao N, Nakai K, Itakura T: Updating of neuronavigation based on images intraoperatively acquired with a mobile computerized tomographic scanner: technical note. Minim Invasive Neurosurg 2003;46:117120.

32 Keles GE, Lamborn KR, Berger MS: Coregistration accuracy and detection of brain shift using intraoperative sononavigation during resection of hemispheric tumors. Neurosurgery 2003;53:556-562.

33 Lindseth F, Kaspersen JH, Ommedal S, Lango T, Bang J, Hokland J, Unsgaard G, Hernes TA: Multimodal image fusion in ultrasoundbased neuronavigation: improving overview and interpretation by integrating preoperative MRI with 3D ultrasound. Comput Aided Surg 2003;8:49-69. 